\title{
ON TEICHMÜLLER SPACES OF TORI
}

\author{
TUOMAS SORVALI
}

1. Introduction. Teichmüller's theorem states that given a sensepreserving homeomorphism $f: S \rightarrow S^{\prime}$ between two compact Riemann surfaces of genus $s>0$, there are in the homotopy class $[f]$ of $f$ extremal mappings $f_{0}$ which minimize the maximal dilatation $K_{f^{\prime}}$ for all $f^{\prime} \in[f]$. The number $\log K_{f_{0}}$ is the Teichmüller distance between the Riemann surfaces $S$ and $S^{\prime}$, relative to the homotopy class of $f$.

A Riemann surface $S$ of genus $s=1$ has the finite complex plane as a universal covering surface, whereas the upper half-plane serves as a universal covering surface if $s>1$. In the first case the euclidean, in the second the non-euclidean metric of the universal covering surface induces a metric in $S$.

For a closed path $\gamma$ on $S$, let $l[\gamma]$ denote the length of the shortest path in the free homotopy class $[\gamma]$. Let $a_{0}$ be the smallest of the numbers $a \geq 1$ such that

$$
l[\gamma] / a \leq l[f \circ \gamma] \leq a l[\gamma]
$$

for all closed paths $\gamma$ on $S$. Then $a_{0}$ is the same for all homeomorphisms homotopic to $f$. Hence $\log a_{0}$ is also a measure for the distance of $S$ and $S^{\prime}$, relative to the homotopy class of $f$.

We shall show that the metrics defined by $\log K_{f_{0}}$ and $\log a_{0}$ are equivalent for tori, i.e. surfaces of genus $s=1$. In the general case the equivalence of these metrics remains unsolved.

2. The Teichmüller space of a torus. Denote by $C$ the finite complex plane and let $G(\omega)$ be the group acting on $C$ which is generated by the translations $z \mapsto z+1$ and $z \mapsto z+\omega$. Then every torus $S$ admits a representation as a quotient space $C / G(\omega)$ for some $\omega \in H=\{z \mid \operatorname{Im} z>0\}$. The number $\omega$ is not uniquely determined by $S$. However, if $S=C / G(\omega)=C / G(\tilde{\omega})$, then there are integers $a, b, c, d$ such that $a d-b c=1$ and $\widetilde{\omega}=(a \omega+b) /$ $(c \omega+d)$. 
Let $f$ be a sense-preserving homeomorphism between the tori $S=C / G(\omega)$ and $S^{\prime}$. Then there is a representation $S^{\prime}=C / G\left(\omega^{\prime}\right)$ of $S^{\prime}$ and a lifting $\tilde{f}: C \rightarrow C$ of $f$ such that $\widetilde{f}(0)=0, \widetilde{f}(1)=1$ and $\widetilde{f}(\omega)=\omega^{\prime}$. The number $\omega^{\prime} \in H$ depends only on $\omega$ and the homotopy class [f] of $f$. If $[f]$ contains conformal mappings, then $\omega^{\prime}=\omega$. We denote by $\omega_{f}$ the number $\omega^{\prime}$ determined by $[f]$.

Let $S_{0}=C / G\left(\omega_{0}\right)$ be a fixed torus and consider all pairs $(S, f)$ where $S$ is a torus and $f: S_{0} \rightarrow S$ a sense-preserving homeomorphism. Two pairs $(S, f)$ and $\left(S^{\prime}, f^{\prime}\right)$ are considered equivalent if $\left[f^{\prime} \circ f^{-1}\right]$ contains a conformal mapping. The space $T\left(S_{0}\right)$ of the equivalence classes $[S, f]$ is the Teichmüller space of $S_{0}$. The Teichmüller distance between the pairs $\left[S_{1}, f_{1}\right]$ and $\left[S_{2}, f_{2}\right]$ is given by

$$
\mu\left(\left[S_{1}, f_{1}\right],\left[S_{2}, f_{2}\right]\right)=\log \inf \left\{K_{f} \mid f \in\left[f_{2} \circ f_{1}^{-1}\right]\right\},
$$

where $K_{f}$ is the maximal dilatation of $f$.

Denote by $h$ the non-euclidean metric in $H$ defined by $d h=(\operatorname{Im} z)^{-1}|d z|$. Then the non-euclidean distance between the points $z_{1}, z_{2} \in H$ is

$$
h\left(z_{1}, z_{2}\right)=\log \frac{\left|z_{1}-\bar{z}_{2}\right|+\left|z_{1}-z_{2}\right|}{\left|z_{1}-\bar{z}_{2}\right|-\left|z_{1}-z_{2}\right|} .
$$

By Teichmüller [2], the $\mu$-distance between the pairs $\left[S_{1}, f_{1}\right]$ and $\left[S_{2}, f_{2}\right]$ is equal to the non-euclidean distance of the points $\omega_{f_{1}}$ and $\omega_{f_{2}}$ :

$$
\mu\left(\left[S_{1}, f_{1}\right],\left[S_{2}, f_{2}\right]\right)=h\left(\omega_{f_{1}}, \omega_{f_{2}}\right) .
$$

3. The dilatation of an isomorphism. Let $f: S \rightarrow S^{\prime}$ be a sense-preserving homeomorphism between the tori $S=C / G(\omega)$ and $S^{\prime}=C / G\left(\omega^{\prime}\right), \omega^{\prime}=\omega_{f}$. If $\widetilde{f}$ denotes the lifting of $f$ for which $\widetilde{f}(0)=0, \widetilde{f}(1)=1$ and $\widetilde{f}(\omega)=\omega^{\prime}$, then

$$
\widetilde{f}(z+m+n \omega)=\widetilde{f}(z)+m+n \omega^{\prime}
$$

for all $z \in C$ and $m, n \in Z$. ( $Z$ is the set of the integers.) Hence $f$ induces an isomorphism $f_{*}: G(\omega) \rightarrow G\left(\omega^{\prime}\right)$ such that

$$
\tilde{f} \circ g=f_{*}(g) \circ \tilde{f}
$$

for all $g \in G(\omega)$.

Let $A\left(f_{*}\right)$ be the set of all numbers $a \geq 1$ for which

$$
|g(0)| / a \leq\left|f_{*}(g)(0)\right| \leq a|g(0)|
$$

for all $g \in G(\omega)$. We call $\delta\left(f_{*}\right)=\min a, a \in A\left(f_{*}\right)$, the dilatation of $f_{*}$. Hence $\delta\left(f_{*}\right)$ is the smallest of the numbers $a \geq 1$ for which 


$$
1 / a \leq\left|\frac{m+n \omega}{m+n \omega^{\prime}}\right| \leq a
$$

for all $m, n \in Z$.

We show that $\delta\left(f_{*}\right)$ is the same as the number $a_{0}$ defined in the introduction. Let $\gamma$ be a closed path on $S$ and $\tilde{\gamma}:[0,1] \rightarrow C$ a lifting of $\gamma$. Then there is a unique $g \in G(\omega)$ such that $g(\tilde{\gamma}(0))=\widetilde{\gamma}(1)$. A closed path $\gamma^{\prime}$ is in the free homotopy class $[\gamma]$ if and only if it has a lifting $\tilde{\gamma}^{\prime}$ such that $g\left(\tilde{\gamma}^{\prime}(0)\right)=$ $\tilde{\gamma}^{\prime}(1)$. Hence $|g(0)|=|\tilde{\gamma}(0)-\tilde{\gamma}(1)|=l[\gamma]$. Similarly $l[f \circ \gamma]=\left|f_{*}(g)(0)\right|$. This proves the assertion.

In [1] we introduced the dilatation of an isomorphism between Fuchsian groups. The definition can be stated as follows. Let $G$ and $G^{\prime}$ be Fuchsian groups acting on $H$. If $g \in G \cup G^{\prime}$, then the translation length of $g$ is defined by

$$
l(g)=\inf \{h(z, g(z)) \mid z \in H\} .
$$

For an isomorphism $j: G \rightarrow G^{\prime}$, let $A(j)$ be the set of the numbers $a \geq 1$ such that

$$
l(g) / a \leq l(j(g)) \leq a l(g)
$$

for all $g \in G$. Then $\delta(j)=\min a, a \in A(j)$, is the dilatation of $j$.

If $g$ is any translation, then

$$
|g(z)-z|=|g(0)|
$$

for all $z \in C$. Hence $|g(0)|$ is an analogue of the translation length of an element of a Fuchsian group, and the definition of $\delta\left(f_{*}\right)$ is obtained from the definition of $\delta(j)$ if $h$ is replaced by the euclidean metric.

4. The dilatation metric in $T\left(S_{0}\right)$. We define in $T\left(S_{0}\right)$ a metric by setting

$$
d\left(\left[S_{1}, f_{1}\right],\left[S_{2}, f_{2}\right]\right)=\log \delta\left(\left(f_{2^{\circ}} f_{1}^{-1}\right)_{*}\right) .
$$

We show next that this dilatation metric $d$ and the Teichmüller metric $\mu$ are equivalent.

In [1] we considered the dilatation metric in the case of the Teichmüller space of a Fuchsian group and showed that distances under this metric are less or equal to distances under the Teichmüller metric. The equivalence of these metrics in this general case is an open question.

$\mathrm{Th}$ e o r e m 1. Let $f: S_{1} \rightarrow S_{2}$ be a sense-preserving homeomorphism between the tori $S_{1}=C / G(\omega)$ and $S_{2}=C / G\left(\omega^{\prime}\right), \omega^{\prime}=\omega_{f}$. If $\omega=x+i y$ and $\omega^{\prime}=x^{\prime}+i y^{\prime}$, then 
(4)

$$
\begin{gathered}
\delta\left(f_{*}\right)=\frac{\left|\omega-\bar{\omega}^{\prime}\right|+\left|\omega-\omega^{\prime}\right|}{y+y^{\prime}-\left|y-y^{\prime}\right|}= \\
\max \left\{\left(2 y^{\prime}\right)^{-1}\left(\left|\omega-\bar{\omega}^{\prime}\right|+\left|\omega-\omega^{\prime}\right|\right), 2 y^{\prime}\left(\left|\omega-\bar{\omega}^{\prime}\right|-\left|\omega-\omega^{\prime}\right|\right)^{-1}\right\} .
\end{gathered}
$$

Proof. Denote by $\bar{R}$ the extended real axis $R \cup\{\infty\}$ and let

$$
M=\max _{x \in \bar{R}}\left|\frac{x+\omega}{x+\omega^{\prime}}\right|, m=\min _{x \in \bar{R}}\left|\frac{x+\omega}{x+\omega^{\prime}}\right| .
$$

Then by (3)

$$
\delta\left(f_{*}\right)=\max (M, 1 / m) .
$$

To calculate $M$ and $m$, we consider the Möbius transformation

$$
B: z \mapsto \frac{z+\omega}{z+\omega^{\prime}} .
$$

Since $B\left(-\omega^{\prime}\right)=\infty, B$ maps $R$ onto a circle $K=B(\bar{R})$ centered at $B\left(-\bar{\omega}^{\prime}\right)=\left(\omega-\bar{\omega}^{\prime}\right) /\left(\omega^{\prime}-\bar{\omega}^{\prime}\right)$. Since $B(\infty)=1$, the radius of $K$ is

$$
\left|\frac{\omega-\bar{\omega}^{\prime}}{\omega^{\prime}-\bar{\omega}^{\prime}}-1\right|=\left|\frac{\omega-\omega^{\prime}}{\omega^{\prime}-\bar{\omega}^{\prime}}\right| \text {. }
$$

Because $B\left(y\left(x^{\prime}-x\right) /\left(y^{\prime}-y\right)-x\right)=y / y^{\prime}$, the circle $K$ intersects the real axis at the points 1 and $y / y^{\prime}>0$. Therefore 0 lies outside $K$, and

$$
M=\left|\frac{\omega-\bar{\omega}^{\prime}}{\omega^{\prime}-\bar{\omega}^{\prime}}\right|+\left|\frac{\omega-\omega^{\prime}}{\omega^{\prime}-\bar{\omega}^{\prime}}\right|=\frac{\left|\omega-\bar{\omega}^{\prime}\right|+\left|\omega-\omega^{\prime}\right|}{\left|\omega^{\prime}-\bar{\omega}^{\prime}\right|},
$$

$$
m=\left|\frac{\omega-\bar{\omega}^{\prime}}{\omega^{\prime}-\bar{\omega}^{\prime}}\right|-\left|\frac{\omega-\omega^{\prime}}{\omega^{\prime}-\bar{\omega}^{\prime}}\right|=\frac{\left|\omega-\bar{\omega}^{\prime}\right|-\left|\omega-\omega^{\prime}\right|}{\left|\omega^{\prime}-\bar{\omega}^{\prime}\right|} .
$$

This proves the latter part of (4).

Since

$$
\frac{\left|\omega^{\prime}-\bar{\omega}^{\prime}\right||\omega-\bar{\omega}|}{\left|\omega-\bar{\omega}^{\prime}\right|^{2}-\left|\omega-\omega^{\prime}\right|^{2}}=1,
$$

we have

$$
1 / m=\frac{\left|\omega^{\prime}-\bar{\omega}^{\prime}\right|}{\left|\omega-\bar{\omega}^{\prime}\right|-\left|\omega-\omega^{\prime}\right|}=\frac{\left|\omega-\bar{\omega}^{\prime}\right|+\left|\omega-\omega^{\prime}\right|}{|\omega-\bar{\omega}|} .
$$

The first part of (4) follows now from

$$
\min \left(2 y, 2 y^{\prime}\right)=y+y^{\prime}-\left|y-y^{\prime}\right| \text {. }
$$


Let $\left[S_{i}, f_{i}\right], i=1,2$, be two points in $T\left(S_{0}\right)$. Denote $\omega=\omega_{f_{1}}, \omega^{\prime}=\omega_{f_{2}}$ and $f=f_{2} \circ f_{1}^{-1}$. Then by Theorem 1 ,

$$
d\left(\left[S_{1}, f_{1}\right],\left[S_{2}, f_{2}\right]\right)=\log \delta\left(f_{*}\right)=\log \max (M, 1 / m),
$$

where $M$ and $m$ are defined by (5).

On the other hand, the numbers $M$ and $m$ determine also the Teichmüller distance of points $\left[S_{1}, f_{1}\right]$ and $\left[S_{2}, f_{2}\right]$. By $(1)$ and $(6), h\left(\omega, \omega^{\prime}\right)=$ $\log (M / m)$. Hence by (2)

$$
\mu\left(\left[S_{1}, f_{1}\right],\left[S_{2}, f_{2}\right]\right)=\log (M / m) .
$$

Theorem 2. The metrics $\mu$ and $d$ of the Teichmüller space $T\left(S_{0}\right)$ satisfy

$$
d\left(\left[S_{1}, f_{1}\right],\left[S_{2}, f_{2}\right]\right) \leq \mu\left(\left[S_{1}, f_{1}\right],\left[S_{2}, f_{2}\right]\right) \leq 2 d\left(\left[S_{1}, f_{1}\right],\left[S_{2}, f_{2}\right]\right)
$$

The inequality (10) is sharp.

Proof. Since $M \geq 1$ and $m \leq 1$,

$$
\max (M, 1 / m) \leq M / m \leq(\max (M, 1 / m))^{2} .
$$

The inequality (10) follows now from (8) and (9).

If we choose $\omega$ and $\omega^{\prime}$ such that $\operatorname{Im} \omega=\operatorname{Im} \omega^{\prime}$, then by (6) and (7) $M=1 / m$. Hence $M / m=(\max (M, 1 / m))^{2}$ and $\mu\left(\left[S_{1}, f_{1}\right], \quad\left[S_{2}, f_{2}\right]\right)=$ $2 d\left(\left[S_{1}, f_{1}\right],\left[S_{2}, f_{2}\right]\right)$.

On the other hand, choose $\operatorname{Re} \omega=\operatorname{Re} \omega^{\prime}$. If $\operatorname{Im} \omega^{\prime}<\operatorname{Im} \omega$, then $m=1$ by (6). Similarly, if $\operatorname{Im} \omega<\operatorname{Im} \omega^{\prime}$, then $M=1$. In both cases $\max (M, 1 / m)=M / m$.

\section{References}

[1] SoRval, T, T.: The boundary mapping induced by an isomorphism of covering groups. - Ann. Acad. Sci. Fenn. Ser. A I 526, 1972, 1-31.

[2] TEICHMÜLLER, O.: Extremale quasikonforme Abbildungen und quadratische Differentiale. - Abh. Preuss. Akad. Wiss. Math.-Natur. Kl. Nr. 22, $1940,1-197$.

University of Helsinki

Department of Mathematics

SF-00100 Helsinki 10

Finland

Received 5 September 1974 